\title{
EXISTENCE RESULTS FOR SUPERLINEAR SEMIPOSITONE BVP'S
}

\author{
V. ANURADHA, D. D. HAI, AND R. SHIVAJI
}

(Communicated by Hal Smith)

Abstract. We consider the existence of positive solutions to the BVP

$$
\begin{gathered}
\left(p(t) u^{\prime}\right)^{\prime}+\lambda f(t, u)=0, \quad r<t<R, \\
a u(r)-b p(r) u^{\prime}(r)=0, \\
c u(R)+d p(R) u^{\prime}(R)=0,
\end{gathered}
$$

where $\lambda>0$. Our results extend some of the existing literature on superlinear semipositone problems and singular BVPs. Our proofs are quite simple and are based on fixed point theorems in a cone.

\section{INTRODUCTION}

We consider the existence of positive solutions for the Sturm-Liouville boundary value problem

$$
\begin{gathered}
\left(p(t) u^{\prime}\right)^{\prime}+\lambda f(t, u)=0, \quad r<t<R \\
a u(r)-b p(r) u^{\prime}(r)=0, \\
c u(R)+d p(R) u^{\prime}(R)=0
\end{gathered}
$$

where $f(t, 0)$ need not be non-negative. The problem (1.1) with Dirichlet boundary conditions was treated by Garaizar [5] and Castro-Shivaji [3]. In [5], the existence of a positive solution to (1.1) for $\lambda>0$ small was established with $p(t)=t^{n-1}$, $f(t, u)=t^{n-1} g(u), g(0)<0$ and $g(u)=O\left(u^{k}\right)$ for some $k>1$. The existence of a unique positive solution to (1.1) for $\lambda>0$ small was proved in [3] with $p(t)=1, f$ independent of $t, f(0)<0, f^{\prime}>0, f^{\prime \prime}>0$ and $f$ superlinear. Further, AnuradhaShivaji [1] extended this existence result for the Robin boundary condition case. In this paper, we shall establish the existence of a positive solution to (1.1) for $\lambda>0$ small under the following general conditions:

$$
f(t, u) \geq-M
$$

for some $M>0$, and

$$
\lim _{u \rightarrow \infty} \frac{f(t, u)}{u}=\infty
$$

uniformly on a compact subinterval of $(r, R)$.

Received by the editors June 10, 1994.

1991 Mathematics Subject Classification. Primary 34B15.

The third author was partially supported by NSF Grants DMS-9215027. This author also thanks the CDSNS at Georgia Institute of Technology, Atlanta, GA, for providing a Visiting Research Scientist position (Fall 1993) during which time this work was completed. 
We also study the problem (1.1) with $f$ possibly singular. Here we do not assume that $f(t, u)$ is decreasing in $u$, thus extending the corresponding results in $[2,4,6,9]$. Our proofs are quite simple and are based on fixed point theorems in a cone.

\section{Existence With $f$ Regular}

We make the following assumptions:

(A.1) $p \in C[r, R], p(t)>0$ for every $t \in[r, R]$.

(A.2) $a, b, c, d \geq 0$ and $a c+a d+b c>0$.

(A.3) $f:[r, R] \times[0, \infty) \rightarrow \mathbb{R}$ is continuous and there exists an $M>0$ such that $f(t, u) \geq-M$ for every $t \in[r, R], u \geq 0$.

(A.4) $\lim _{u \rightarrow \infty} \frac{f(t, u)}{u}=\infty$ uniformly on a compact subinterval $[\alpha, \beta]$ of $(r, R)$.

Then we have

Theorem 2.1. Let (A.1)-(A.4) hold. Then the problem (1.1) has a positive solution for $\lambda>0$ sufficiently small.

In order to prove Theorem 2.1, we first recall:

Theorem A ([7]-[8]). Let $\mathbb{K}$ be a cone in a Banach space $E$ and let $A: \mathbb{K} \rightarrow \mathbb{K}$ be a completely continuous operator. Let $0<r<R$ be such that

(i) $u \leq A u \Rightarrow\|u\| \neq r$,

(ii) $u \geq A u \Rightarrow\|u\| \neq R$.

Here $u \leq v$ iff $v-u \in \mathbb{K}$. Then $A$ has a fixed point $u$ with $r<\|u\|<R$.

We further need the following lemmas.

Lemma 2.1. Let (A.1), (A.2) hold and let $u$ satisfy

$$
\begin{gathered}
\left(p(t) u^{\prime}\right)^{\prime}=-v, \quad r<t<R, \\
a u(r)-b p(r) u^{\prime}(r)=0, \quad c u(R)+d p(R) u^{\prime}(R)=0,
\end{gathered}
$$

where $v \in L^{1}(r, R), v \geq 0$. Then

$$
u(t) \geq|u|_{0} q(t), \quad t \in[r, R]
$$

where

$$
q(t)=\min \left(\frac{b+a \int_{r}^{t} \frac{1}{p}}{b+a \int_{r}^{R} \frac{1}{p}}, \frac{d+c \int_{t}^{R} \frac{1}{p}}{d+c \int_{r}^{R} \frac{1}{p}}\right) .
$$

Here $|\cdot|_{0}$ stands for the sup norm.

Proof of Lemma 2.1. It can be verified that

where

$$
u(t)=\int_{r}^{R} K(t, s) v(s) d s, \quad t \in[r, R],
$$

$$
K(t, s)= \begin{cases}\alpha^{-1}\left(b+a \int_{r}^{s} \frac{1}{p}\right)\left(d+c \int_{t}^{R} \frac{1}{p}\right) & \text { if } s \leq t, \\ \alpha^{-1}\left(b+a \int_{r}^{t} \frac{1}{p}\right)\left(d+c \int_{s}^{R} \frac{1}{p}\right) & \text { if } s \geq t,\end{cases}
$$

where $\alpha=a d+a c \int_{r}^{R} \frac{1}{p}+b c$. 
Let $|u|_{0}=u\left(t_{0}\right)$ for some $t_{0} \in[r, R]$. We verify that

$$
\frac{K(t, s)}{K\left(t_{0}, s\right)} \geq q(t), \quad s, t, t_{0} \in(r, R)
$$

Indeed, if $t, t_{0} \leq s$,

$$
\frac{K(t, s)}{K\left(t_{0}, s\right)}=\frac{b+a \int_{r}^{t} \frac{1}{p}}{b+a \int_{r}^{t_{0}} \frac{1}{p}} \geq \frac{b+a \int_{r}^{t} \frac{1}{p}}{b+a \int_{r}^{R} \frac{1}{p}}
$$

if $t \leq s \leq t_{0}$,

$$
\frac{K(t, s)}{K\left(t_{0}, s\right)}=\frac{\left(b+a \int_{r}^{t} \frac{1}{p}\right)\left(d+c \int_{s}^{R} \frac{1}{p}\right)}{\left(b+a \int_{r}^{s} \frac{1}{p}\right)\left(d+c \int_{t_{0}}^{R} \frac{1}{p}\right)} \geq \frac{\left(b+a \int_{r}^{t} \frac{1}{p}\right)}{\left(b+a \int_{r}^{R} \frac{1}{p}\right)} .
$$

The other cases are treated similarly. Thus

$$
u(t)=\int_{r}^{R} \frac{K(t, s)}{K\left(t_{0}, s\right)} K\left(t_{0}, s\right) v(s) d s \geq|u|_{0} q(t), \quad t \in[r, R] .
$$

Lemma 2.2. Let (A.1), (A.2) hold and let $\bar{w}$ be the solution of

$$
\begin{gathered}
\left(p(t) u^{\prime}\right)^{\prime}=-1, \quad r<t<R, \\
a u(r)-b p(r) u^{\prime}(r)=0, \\
c u(R)+d p(R) u^{\prime}(R)=0 .
\end{gathered}
$$

Then there exists a positive number $C$ such that $\bar{w}(t) \leq C q(t)$ for every $t \in[r, R]$.

Proof of Lemma 2.2. We have

$$
\begin{aligned}
\bar{w}(t)= & \alpha^{-1}\left[\left(d+c \int_{t}^{R} \frac{1}{p}\right)\left(\int_{r}^{t}\left(b+a \int_{r}^{s} \frac{1}{p}\right) d s\right)\right. \\
& \left.+\left(b+a \int_{r}^{t} \frac{1}{p}\right)\left(\int_{t}^{R}\left(d+c \int_{s}^{R} \frac{1}{p}\right) d s\right)\right] \\
\leq & \alpha^{-1}(R-r)\left(b+a \int_{r}^{t} \frac{1}{p}\right)\left(d+c \int_{t}^{R} \frac{1}{p}\right) \leq C q(t),
\end{aligned}
$$

where $C=\alpha^{-1}\left(b+a \int_{r}^{R} \frac{1}{p}\right)\left(d+c \int_{r}^{R} \frac{1}{p}\right)(R-r)$.

Proof of Theorem 2.1. Let $\lambda$ satisfy

$$
0<\lambda<\min \left(\frac{1}{C_{1}|\bar{w}|_{0}}, \frac{1}{C M}\right),
$$

where $C_{1}=\sup _{r \leq s \leq R, 0 \leq t \leq 1} g(s, t), g(s, t)=f(s, t)+M$ and $C$ is the constant defined in Lemma 2.2 . 
Let $w=\lambda M \bar{w}$. Then $u$ is a positive solution of (1.1) iff $\tilde{u}=u+w$ is a solution of

$$
\begin{gathered}
\left(p(t) u^{\prime}\right)^{\prime}=-\lambda \tilde{g}(t, u-w), \\
a u(r)-b p(r) u^{\prime}(r)=0, \\
c u(R)+d p(R) u^{\prime}(R)=0
\end{gathered}
$$

with $\tilde{u}(t)>w(t)$ on $(r, R)$. Here $\tilde{g}(t, u)=g(t, u)$ for $u \geq 0$, and $g(t, u)=g(t, 0)$ for $u<0$.

Let $\mathbb{K}=\left\{u \in C[r, R]: u(t) \geq|u|_{0} q(t), t \in[r, R]\right\}$, where $q$ is defined by Lemma 2.1. For each $v \in \mathbb{K}$, let $u=A v$ be the solution of

$$
\begin{gathered}
\left(p(t) u^{\prime}\right)^{\prime}=-\lambda \tilde{g}(t, v-w), \\
a u(r)-b p(r) u^{\prime}(r)=0, \\
c u(R)+d p(R) u^{\prime}(R)=0 .
\end{gathered}
$$

By Lemma 2.1, $A: \mathbb{K} \rightarrow \mathbb{K}$ and it can be verified that $A$ is completely continuous. We shall prove that $A$ has a fixed point in $\mathbb{K}$ by using Theorem A [7]. Let $u \in \mathbb{K}$ be such that $u \leq A u$. We claim that $|u|_{0} \neq 1$. Indeed, if $|u|_{0}=1$, then we have

$$
u(t) \leq \lambda \int_{r}^{R} K(t, s) \tilde{g}(s, u-w) d s \leq \lambda C_{1} \bar{w}(t), \quad t \in[r, R],
$$

where $K(t, s)$ is given by (2.1). This implies

$$
1 \leq \lambda C_{1}|\bar{w}|_{0},
$$

a contradiction to (2.2), proving the claim.

Now, let $u \in \mathbb{K}$ with $u \geq A u$. Then we have

$$
u(t) \geq \lambda \int_{\alpha}^{\beta} K(t, s) \tilde{g}(s, u-w) d s .
$$

Let $\widetilde{M}>0$ and let $|u|_{0}=\bar{R}$. Since

$$
w(s)=\lambda M \bar{w}(s) \leq \frac{\lambda C M}{\bar{R}} u(s),
$$

it follows that

$$
u(s)-w(s) \geq\left(1-\frac{\lambda C M}{\bar{R}}\right) u(s) .
$$

Therefore if $\bar{R}$ is sufficiently large, we have

$$
u(s)-w(s) \geq \frac{1}{2} u(s) \geq \frac{1}{2} \bar{R} \delta, \quad s \in[\alpha, \beta],
$$

where $\delta=\min _{\alpha \leq s \leq \beta} q(s)$, and

$$
\tilde{g}(s, u-w)=g(s, u-w) \geq \widetilde{M}(u(s)-w(s)) \geq \frac{\widetilde{M} \bar{R} \delta}{2}
$$

by (A.4).

Combining (2.3)-(2.5), we obtain

$$
\bar{R} \geq \frac{\lambda \widetilde{M} \bar{R} \delta}{2}\left(\sup _{r \leq t \leq R} \int_{\alpha}^{\beta} K(t, s) d s\right),
$$


which is a contradiction if $\widetilde{M}$ is sufficiently large. So there exists an $\bar{R}>1$ such that $|u|_{0} \neq \bar{R}$. By Theorem A (expansion theorem), $A$ has a fixed point $\tilde{u}$ with $1 \leq|\tilde{u}|_{0} \leq \bar{R}$.

It follows that $\tilde{u}(t) \geq q(t) \geq \lambda C M q(t) \geq w(t)$, and so $u=\tilde{u}-w$ is a positive solution to (1.1), completing the proof of Theorem 2.1.

\section{EXISTENCE With $f$ Singular}

We now turn our attention to the problem (1.1) with $f$ possibly singular. We make the following assumptions:

(A.5) $f:(r, R) \times(0, \infty) \rightarrow(0, \infty)$ is continuous.

(A.6) There exist positive constants $C, \alpha, \beta$ with $r<\alpha<\beta<R$, and $h \in L^{1}(\alpha, \beta)$, $h \geq 0, h \neq \equiv$ such that

$$
f(t, u) \geq h(t)
$$

for $t \in(\alpha, \beta), u \leq C$.

(A.7) For each $\theta>0$, there exists $p_{\theta} \in L^{1}(r, R)$ such that

$$
f(t, u) \leq p_{\theta}(t), \quad t \in(r, R),
$$

for every $u \in C[r, R]$ with $u(t) \geq \theta q(t)$, where $q(t)$ is given by Lemma 2.1.

We then have

Theorem 3.1. Let (A.1), (A.2), (A.5)-(A.7) hold, and let $\lambda>0$; then the problem (1.1) has a positive solution $u \in C^{1}[r, R] \cap C^{2}(r, R)$.

In order to prove Theorem 3.1 we first recall:

Theorem $\mathbf{B}([7]-[8])$. Let $\mathbb{K}$ be a cone in a Banach space $E$,

$$
D=\{u \in \mathbb{K}: r \leq\|u\| \leq R\}
$$

and $A: D \rightarrow \mathbb{K}$ be a completely continuous operator such that

(i) $u \in D, \lambda \in(0,1), u=\lambda A u \Rightarrow\|u\| \neq R$,

(ii) $u \in D, \lambda>1, u=\lambda A u \Rightarrow\|u\| \neq r$,

(iii) $\inf _{\|u\|=r}\|A u\|>0$.

Then $A$ has a fixed point in $D$.

Proof of Theorem 2.1. Let $\mathbb{K}$ be the cone as in the proof of Theorem 2.1 and let

$$
\begin{aligned}
& r_{0}=\frac{1}{2} \min \left(\lambda \sup _{r \leq t \leq R} \int_{\alpha}^{\beta} K(t, s) h(s) d s, C, 1\right), \\
& R_{0}=2 \max \left(\lambda \sup _{r \leq t \leq R} \int_{r}^{R} K(t, s) p_{1}(s) d s, 1\right),
\end{aligned}
$$

where $K(t, s)$ is defined by $(2.1)$.

Let $D=\left\{u \in \mathbb{K}: r_{0} \leq|u|_{0} \leq R_{0}\right\}$. For each $v \in D$, let $u=A v$ be the solution of

$$
\begin{gathered}
\left(p(t) u^{\prime}\right)^{\prime}=-\lambda f(t, v), \\
a u(r)-b p(r) u^{\prime}(r)=0, \\
c u(R)+d p(R) u^{\prime}(R)=0 .
\end{gathered}
$$


Note that $u$ exists since $f(t, v) \leq p_{r_{0}}(t)$, by (A.7). By Lemma 2.1, $A: D \rightarrow \mathbb{K}$ and it can be verified that $A$ is completely continuous. To apply Theorem $\mathrm{B}$, it is sufficient to verify that

$$
\begin{aligned}
|A u|_{0}>r_{0} & \text { for } u \in D \text { with }|u|_{0}=r_{0}, \\
|A u|_{0}<R_{0} & \text { for } u \in D \text { with }|u|_{0}=R_{0} .
\end{aligned}
$$

Let $u \in D$ with $|u|_{0}=r_{0}$. Then by (A.6)

$$
A u(t)=\lambda \int_{r}^{R} K(t, s) f(s, u) d s \geq \lambda \int_{\alpha}^{\beta} K(t, s) h(s) d s, \quad t \in[r, R],
$$

and so $|A u|_{0}>r_{0}$.

Next, let $u \in D$ with $|u|_{0}=R_{0}$. Then $u(t) \geq q(t)$ and by (A.7) we have

$$
A u(t) \leq \lambda \int_{r}^{R} K(t, s) p_{1}(s) d s
$$

and so $|A u|_{0}<R_{0}$.

Thus $A$ has a fixed point $u$ which is a $C^{1}[r, R] \cap C^{2}(r, R)$ positive solution to (1.1), completing the proof of Theorem 3.1.

Remark 1. Condition (A.7) is satisfied if

$$
\int_{r}^{R} f(t, \theta s(t)) d t<\infty
$$

for every $\theta>0$, where $s(t)=\min (t-r, R-t)$. In the case where $b, d>0,(\mathrm{~A} .7)$ is equivalent to

$$
\int_{r}^{R} f(t, u) d t<\infty
$$

for every $u>0$.

Remark 2. In the case where $p(t)=1$, the existence of a positive $C^{1}[r, R] \cap C^{2}(r, R)$ solution to (1.1) was studied in $[4,6,9]$. The result in $[6]$, which extends the one in [9], requires that $f$ satisfy (A.2), (A.5), (A.8), (A.9), $f(t, u) \rightarrow 0$ as $u \rightarrow \infty$ and $f(t, u) \rightarrow \infty$ as $u \rightarrow 0$ uniformly on compact subsets of $(r, R)$, and $f(t, u)$ be decreasing in $u$ for each $t$. In [4], (A.8) and the limiting conditions of [6] were removed, provided $a, b, c$, and $d$ are positive. Also, the result in [2] when applied to the problem (1.1) with $p(t)=1$ requires that $f(t, u)$ be decreasing in $u$ and $f$ satisfy (A.7). Thus Theorem 3.1 unifies and extends the corresponding results in $[2,4,6,9]$.

\section{REFERENCES}

1. V. Anuradha and R. Shivaji, A quadrature method for classes of multi-parameter two point boundary value problems, Applicable Anal. (to appear).

2. J. V. Baxley, Some singular nonlinear boundary value problems, Siam J. Math. Anal. 22 (1991), 463-479. MR 92f:34017

3. A. Castro and R. Shivaji, Nonnegative solutions for a class of nonpositone problems, Proc. Roy. Soc. Edinburgh Sect. A 108 (1988), 291-302. MR 90m:34040

4. A. M. Fink, J. A. Gatica, G. E. Hernendez, and P. Waltman, Approixmation of solutions of singular second order boundary value problems, Siam. J. Math. Anal. 22 (1991), 440-462. MR 92f:34016

5. X. Garaizar, Existence of positive radical solutions for semilinear elliptic equations in the annulus, J. Differential Equations 70 (1987), 69-92. MR 89f:35019 
6. J. A. Gatica, V. Oliver, and P. Waltman, Singular nonlinear boundary value problems for second order ordinary differential equations, J. Differential Equations 79 (1989), 62-78. MR 90f: 34030

7. G. B. Gustafson and K. Schmitt, Method of nonlinear analysis in the theory of differential equations, Lecture notes, University of Utah, 1975.

8. M. A. Krasnosel'skii, Positive solutions of operator equations, Noordhoff, Gronigen, the Netherlands, 1964. MR 31:6107

9. S. Taliaferro, A nonlinear singular boundary value problem, Nonlinear Anal. 3 (1979), 897904. MR 81i:34011

(V. Anuradha) Department of Mathematics and Statistics, University of Arkansas at Little Rock, Little Rock, Arkansas 72212

(D. D. Hai and R. Shivaji) Department of Mathematics and Statistics, Mississippi State University, Mississippi State, Mississippi 39762

E-mail address, D. D. Hai: dang@math.msstate.edu

E-mail address, R. Shivaji: shivaji@math.msstate.edu 